\title{
BMJ Open Efficacy of brief intervention for drug misuse in primary care facilities: systematic review and meta- analysis protocol
}

\author{
Ethan Sahker (D) , ${ }^{1,2}$ Masatsugu Sakata (D) , ${ }^{1}$ Rie Toyomoto, ${ }^{1}$ Chiyoung Hwang, ${ }^{1,3}$ \\ Kazufumi Yoshida, ${ }^{1}$ Yan Luo, ${ }^{1}$ Norio Watanabe, ${ }^{1}$ Toshi A Furukawa ${ }^{1}$
}

To cite: Sahker E, Sakata M, Toyomoto R, et al. Efficacy of brief intervention for drug misuse in primary care facilities: systematic review and metaanalysis protocol. BMJ Open 2020;10:e036633. doi:10.1136/ bmjopen-2019-036633

- Prepublication history and additional material for this paper are available online. To view please visit the journal (http:// dx.doi.org/10.1136/bmjopen2019-036633).

Received 23 December 2019 Revised 23 July 2020 Accepted 28 July 2020

Check for updates

(C) Author(s) (or their employer(s)) 2020. Re-use permitted under CC BY-NC. No commercial re-use. See rights and permissions. Published by BMJ.

${ }^{1}$ Department of Health Promotion and Human Behavior, Graduate School of Medicine, School of Public Health, Kyoto University, Kyoto, Japan

2Japan Society for the Promotion of Science (JSPS), Overseas Fellowship Division, Kojimachi, Chiyoda-ku, Tokyo, Japan

${ }^{3}$ Japan Society for the Promotion of Science (JSPS), Research Fellowship Division, Kojimachi, Chiyoda-ku, Tokyo, Japan

Correspondence to

Dr Ethan Sahker;

sahker.ethan.2e@kyoto-u.ac.jp

\section{ABSTRACT}

Introduction Drug misuse is associated with significant global morbidity, mortality, economic costs and social costs. Many primary care facilities have integrated drug misuse screening and brief intervention $(\mathrm{BI})$ into their usual care delivery. However, the efficacy of BI for drug misuse in primary care has not been substantiated through metaanalysis. The aim of this systematic review and metaanalysis is to determine the efficacy of $\mathrm{BI}$ for drug misuse in primary care settings.

Methods and analysis We will include all randomised controlled trials comparing primary care-delivered $\mathrm{BI}$ for drug misuse with no intervention or minimal screening/ assessment and usual care. Primary outcomes are (1) drug use frequency scores and (2) severity scores at intermediate follow-up (4-8 months). We will retrieve all studies through searches in CENTRAL, Embase, MEDLINE and PsycINFO until 31 May 2020. The reference list will be supplemented with searches in trial registries (eg, www. clinicaltrials.gov) and through relevant existing study reference lists identified in the literature. We will conduct a random-effect pairwise meta-analysis for primary and secondary outcomes. We will assess statistical heterogeneity though visual inspection of a forest plot and calculate $P$ statistics. We will assess risk of bias using the Cochrane Risk of Bias Tool V.2 and evaluate the certainty of evidence through the Grading of Recommendations Assessment, Development and Evaluation (GRADE) approach. Sensitivity analyses will account for studies with control group variations and studies with a high risk of bias. If heterogeneity is present, subgroup analyses will consider patient variables of age, sex/gender, race/ ethnicity, per cent insured, baseline severity and primary drug misused.

Ethics and dissemination This study will use published aggregate data and will not require ethical approval. Findings will be disseminated in a peer-reviewed journal.

\section{BACKGROUND}

Drug misuse is associated with significant global morbidity, mortality, economic costs and social costs. ${ }^{1}$ Drug misuse is defined by hazardous use of any non-alcohol-related drug (eg, cannabis, opioids and stimulants) beyond legal and medical guidelines. ${ }^{2}$ Drug
Strengths and limitations of this study

- Our systematic review and meta-analysis will be the first to examine the efficacy of brief intervention (BI) for drug misuse in primary care.

- This study will investigate the efficacy of BI for drug use frequency and severity at multiple time points.

- Limitations of individual studies will be judged with the revised Cochrane Risk of Bias Tool V.2.

- This study will not investigate treatment referral and is limited to screening and $\mathrm{BI}$ in primary care.

- Significant heterogeneity will be assessed through subgroup analyses by prespecified patient characteristics.

misuse may increase the risk of harmful consequences in mental, physical and social domains, regardless of any diagnosed substance use disorder (SUD). ${ }^{1}$ Additionally, drug misuse may indicate a need for treatment as part of a diagnosable SUD characterised by risky use, impaired control, social impairment, tolerance or withdrawal for a specific substance. ${ }^{3}$ In 2017 , it was estimated that globally, about $5 \%$ of adults report illicit drug use and $0.4 \%$ fit criteria for a drugrelated SUD. ${ }^{1}$ However, only about $10 \%$ of people with an SUD receive treatment. ${ }^{45}$ Most patients who receive specialised SUD treatment are referred through criminal justice or self-referral sources ${ }^{6}{ }^{6}$ which means that $10 \%$ who receive treatment are likely to be highly motivated or compelled to change. This leaves a $90 \%$ disparity known as the treatment gap. The treatment gap led to using primary care facilities as a method of identifying those who may benefit from treatment.

Those who demonstrate drug misuse are likely to present in primary care facilities, are more likely to be admitted and have higher odds of substance-related health complications than those who do not misuse 
drugs. ${ }^{78}$ However, healthcare professionals historically demonstrate some of the lowest referral rates to SUD treatment. $^{9}{ }^{10}$ Ideally, drug misuse would be universally screened and treated in primary care facilities. While drug misuse screenings have improved, follow-up interventions may still be lacking. For example, a good $75 \%$ of those who see a healthcare provider report being screened for alcohol use. ${ }^{11}$ Unfortunately, when patients do report substance misuse, providers rarely follow-up with advice $(20 \%)$ or SUD treatment referral $(7 \%){ }^{11}$

The current trend of SUD and intervention research focuses on training programmes, counselling and motivational interviewing (MI). ${ }^{12}$ To address the SUD treatment gap and referral inconsistency in primary care, the Substance Abuse and Mental Health Services (SAMHSA) developed and funded a large-scale programme called screening, brief intervention and referral to treatment (SBIRT). ${ }^{13}$ SBIRT identifies patients in primary care who would not normally seek treatment, provides a short motivational intervention and, if needed, refers to outside specialty treatments. Many resources, including millions of dollars and healthcare professional time, have been invested on research and service implementation of SBIRT programmes across the globe. ${ }^{14}$ Cost analysis suggests it is worth the price to initiate patient change behaviours. ${ }^{16}$ However, the significant bodies of research investigating SBIRT findings have been mixed, with supportive findings for alcohol misuse, but lacking evidence for drug misuse. ${ }^{13}$ 17-19 Additionally, there is insufficient evidence to support the efficacy of the SBIRT referral to treatment component due to poor referral follow-ups. ${ }^{20}$ The incongruent evidence for substance misuse outcomes and lack of evidence for referral efficacy is thought to be due to issues in data collection, intervention facilitation uniformity and site biases. ${ }^{21}$ These mixed SBIRT findings suggest focused investigations of the brief intervention (BI) component for drug misuse are required. ${ }^{1922}$

Many primary care facilities have integrated drug misuse screening and BI into their usual care delivery; both SAMHSA-related SBIRT and non-SBIRT programmes. Because many people visit primary care facilities regularly, they are an optimal setting to screen for drug misuse and to follow up with BI on site. BI in primary care targets patients who otherwise may not seek treatment. However, the efficacy of BI for drug misuse was not well substantiated prior to a major SAMHSA-funded BI programme deployment in 2004 and remains unclear over 15 years after its inception. ${ }^{23}$ A systematic review and meta-analysis is necessary to determine BI efficacy for drug misuse in primary care.

\section{Brief intervention}

BI consists of one to five individual sessions lasting 5-60 min each for low to moderate misuse, usually conducted on site as soon as possible. ${ }^{24} \mathrm{~A}$ meta-analysis showed the median BI length is 25 min. ${ }^{25}$ Extended BIs may include additional sessions or a follow-up telephone call of 5-30 min ('booster') to improve motivation after discharge from primary care. ${ }^{18} 26$ A meta-analysis showed the median extended BI length is 100 min over multiple sessions. ${ }^{25} \mathrm{BI}$ can be conducted by physicians, comedical professionals, trained laypersons and through computer programmes. BI is undergirded by a significant number of efficacy and effectiveness studies for alcohol use in many primary care facilities. ${ }^{21}$

BI was developed out of social, cognitive and behavioural approaches to psychotherapy. ${ }^{26}$ In a systematic review and meta-analysis of BI for alcohol misuse, Kaner and colleagues ${ }^{26}$ outlined key BI components to include (1) feedback about personal use and harm, (2) risks of continued use, (3) benefits of reducing one's use, (4) advice on how to reduce one's use, (5) motivational enhancement and (6) developing a plan to reduce use. $\mathrm{BI}$ is grounded in the transtheoretical model of change. ${ }^{27}$ BI uses the FRAMES approach: feedback, responsibility to change, advice, menu of treatment options, empathy and supporting of self-efficacy. ${ }^{18232628}$ In addition, BI relies heavily on $\mathrm{MI}^{29}$ and motivational enhancement therapy (MET),${ }^{30}$ which are directive, client-centred and aimed at resolving ambivalence through provider feedback to elicit behaviour change. MI and MET are both evidence-based treatments for SUDs with strong research support. ${ }^{31}$

$\mathrm{BI}$ in primary care works by first identifying drug misuse through screening. Patients present to primary care facilities seeking medical care. Patients are screened as part of admission/sign-in procedures. If unhealthy substance use is reported, further assessment determines the appropriate level of care, either no treatment, BI or referral to outside specialty treatment. Screenings may consist of a simple prescreen to determine any drug use. If positive, a formal screening would be activated. For example, some programmes use the drug abuse screening test (DAST) ${ }^{3233}$ to screen for drug misuse. The National Institute on Drug Abuse $^{34}$ outlined the DAST-10 as scored from 0 to 10 and divided into zones indicating appropriate intervention levels. Scores from 0 to 2 suggest no/low level problems and may indicate monitoring, feedback or no treatment. Scores of 3-8, moderate/substantial problems, suggest further assessment and BI may be indicated. Scores of 9-10, severe problems, suggest intensive assessment and may indicate referral to specialty treatment providers.

\section{Gap in the knowledge}

BI in primary care is well documented to be effective, but only for reducing alcohol use frequency and severity. 18262835 Screening and BI for alcohol have been given a B GRADE recommendation from the US Preventive Services Task Force. ${ }^{36}$ Additionally, alcohol findings may not generalise to the treatment of other drug misuse. Evidence from BI for drug misuse studies remains equivocal. ${ }^{1718222328353738}$ For instance, a multisite observational study reported significant reductions in drug use outcomes. ${ }^{18}$ Conversely, the US Preventive Services Task Force concluded there was insufficient evidence to assess the risks and benefits of primary care screening 
or BI for drug misuse. ${ }^{39}$ Yet, no meta-analyses have been conducted. Furthermore, patient variables such as sex, ${ }^{18}$ age $^{40}$ and primary problem substance ${ }^{4142}$ moderate drug use outcomes have not been evaluated in a systematic way. In response to a call for further research into drug misuse $\mathrm{BI},{ }^{22}{ }^{43}$ several randomised controlled trials (RCTs) have been completed in the past 10 years, and a meta-analysis would now help to determine BI efficacy in primary care.

\section{Present study}

This study aimed to address the gap in the research base by investigating the efficacy of BI for drug misuse frequency and severity in primary care settings through a pairwise systematic review and meta-analysis. Findings will help to determine if the use of $\mathrm{BI}$ for drug misuse in primary care is warranted or if changes to treatment protocols are needed. We aimed to evaluate the overall efficacy of BI compared with no intervention through (1) drug use frequency at intermediate follow-up (4-8 months) and (2) drug use severity at intermediate follow-up (4-8 months). Secondary outcomes under investigation aimed to discover BI efficacy in terms of frequency and severity at short-term follow-up ( $<4$ months) and longterm follow-up ( $>8$ months). Additionally, outcome comparisons by patient characteristics will be assessed for age, sex/gender, race/ethnicity, insurance and primary problem drug.

\section{METHODS}

We submitted this study protocol to PROSPERO (CRD42020157733). Methods for this systematic review and pairwise meta-analysis follow the Preferred Reporting Items for Systematic Review and Meta-Analysis Protocols. ${ }^{44}$

\section{Criteria for considering studies}

\section{Types of studies}

Studies will be limited to RCTs, including cluster randomised trials, comparing BI with control conditions. Control conditions may be considered usual care, no intervention, minimal screening/assessment or informational pamphlets on drug use. Quasi-randomised trials, RCTs without control conditions and implementation studies only investigating provider adherence or cost effectiveness will be excluded.

\section{Participants}

Participants in the study include people presenting in primary care facilities for physical health concerns who are screened for and report drug misuse. Drug misuse includes legal cannabis, illicit substances, over-thecounter drugs and prescription drugs. Alcohol misuse will not be included. No restrictions will be made in terms of age, sex/gender or country.

\section{Intervention}

We aimed to include BI studies with a range of one to five sessions, with each session lasting from 5 to $60 \mathrm{~min}$. The intervention may be composed of any one treatment or a combination of the following delivery modalities: in-person, phone, mailed letter or computer. The intervention may be facilitated by any healthcare provider of any education level.

\section{Comparators}

We will include 'no intervention' controls consisting of minimal screening/assessment only. No intervention may also be referred to as usual care and may include drug screening and an information pamphlet about drug misuse and self-help resources. Comparators that include more than screening/assessments needed for study inclusion and randomisation, or an information pamphlet, will be included in the main analysis but excluded in a sensitivity analysis.

\section{Outcomes}

Our outcomes are past 30 days' drug use frequency or severity. Primary outcomes are (1) drug use frequency and (2) severity at intermediate follow-up (4-8 months). Secondary outcomes are (1) frequency and (2) severity at short-term follow-up ( $<4$ months) and (3) frequency and (4) severity at long-term follow-up ( $>8$ months). If studies use multiple severity measures, we will prioritise based on the majority to have a more congruent outcome. This will not be necessary for frequency, which is a count of days in the past month, and different measures are on the same metric. Typically, studies report follow-ups in intervals of $3,6,9$ or 12 months. If a study reports two separate follow-ups that both fall within our intermediate range (ie, 4 and 6 months), we will prioritise the follow-up closest to the midpoint (ie, 6 months).

Outcomes include

Frequency (count)-Frequency is measured as the number of days in the past month the primary problem substance was used. Multiple instruments may be appropriate for analysis and will include but will not be limited to the timeline followback (TLFB) ${ }^{45}$ and the Addiction Severity Index (ASI)-Lite Frequency Score. ${ }^{46}$ Severity (continuous) - Severity is measured as a composite score which accounts for past 30 days' use frequency and includes measures of cravings, withdrawal and drug use consequences in physical, psychological, social and vocational domains. Again, multiple instruments may be appropriate for analysis and will include, but will not be limited to, the Alcohol, Smoking and Substance Involvement Screening Test (ASSIST) ${ }^{47}$ and the $\mathrm{ASI}^{46}$ composite scores.

\section{Setting}

Following previous research, primary care is defined as immediately accessible and general care to a broad range of health concerns in the community or hospital settings. ${ }^{25}{ }^{26}$ Examples include emergency departments, family practice, women's health clinics and community clinics. 


\section{Search strategy}

We will search studies in CENTRAL, Embase, MEDLINE and PsycINFO until 31 May 2020. The preliminary search strategy for MEDLINE (see online supplementary appendix 1) will be adapted for other database terms. Searches will be supplemented with trial registries (eg, www.clinicaltrials.gov) and through relevant existing literature review and RCT reference lists. We will not place any restrictions on language, date, document type or publication status for inclusion. We will use keywords drug misuse, intervention, setting and study method. A database search professional will be enlisted and consulted for the initial search. We will evaluate similar systematic and non-systematic reviews to find potentially eligible trials. Search queries, dates and identified records will be made available in the online supplementary appendix 1 and will be represented in a Preferred Reporting Items for Systematic Reviews and Meta-Analyses (PRISMA) flowchart.

\section{Study selection}

Reviewers will independently examine titles and abstracts of studies identified in the initial search. Irrelevant or duplicate reports will be removed. Reviewers will then meet to form a consensus on study inclusions. Next, we will obtain full texts of potentially relevant articles. Teams will independently assess each paper for inclusion per criteria, then consensus will be made on conflicting assessments. Coauthors not involved in selection will supervise and form final judgements if consensus cannot be made. If it is still not possible to determine study eligibility, study authors will be contacted for further information. We will report the inter-rater reliability for study inclusion. Multiple published papers related to the same study will be collated and included in the report. The study selection process will be recorded in detail to produce a PRISMA flowchart.

\section{Data extraction}

Reviewers will independently summarise the study details to include methods, participants, interventions, referral information, comparator interventions, treatment outcomes, dropouts and potential biases using a standardised data extraction form. We will develop and pilot the data extraction form for this review. For inconsistencies, review teams will discuss lacking information. Two senior researchers will provide mediation for reporting discrepancies. Data will be entered into Review Manager V.5 software and all authors will independently check entries for inconsistencies.

\section{Assessment of risk of bias in included studies}

Two review authors will independently assess the risk of bias for each study using the Cochrane Risk of Bias Tool V.2 (RoB 2) ${ }^{48}$ Risk of bias will be rated for each domain at three levels: high, low and unclear. Evidence will be provided by means of a quotation exemplar. Evidence and justification for judgement of risk of bias will be presented in a table. Studies providing few or no details about randomisation or blinding will be contacted for clarification. We will evaluate studies providing outcomes on bias due to randomisation process, deviations from intended interventions, missing outcomes, measurement of outcome and selection of reported results. ${ }^{48}$ We will report the inter-rater reliability for assessment of bias.

Study classification per RoB $2^{48}$ are as follows:

Low risk of bias: All domains are rated at low risk of bias.

Some concerns: At least one domain is rated as concerning, and no domain is rated at high risk of bias.

High risk of bias: At least one domain is rated to be at high risk of bias, or multiple domains are concerning.

\section{Measures of treatment effect}

Data synthesis

For studies assessing drug use frequency on the same outcome metric (eg, days used per TLFB or ASI), we will calculate the mean difference (MD) with $95 \%$ CIs. Because different instruments measure the same count metric, they can be combined and compared with MD. For studies assessing drug use severity using different outcome measures (eg, severity per ASI or ASSIST), we will calculate the standardised MD with $95 \%$ CIs. The SMD is interpreted as small effect (0.2), moderate effect $(0.5)$ and large effect (0.8). ${ }^{49}$ ASI and ASSIST have been shown to have strong concurrent validity $(\mathrm{r}=0.73-0.82, \mathrm{p}<0.001)$ for derived composite severity scores. ${ }^{50-52}$ Studies with more than two treatment arms can cause problems in pairwise meta-analysis. When multiple arms are reported in a single study, we will include only relevant arms. If two treatment arms are relevant, the control will be split and compared with each arm separately. ${ }^{483}$

We will analyse results from cluster $\mathrm{RCTs}^{48}$ by taking account of intracluster correlation coefficients (ICCs). When the ICC is not reported, we will contact the authors. If the ICC is unavailable, we will use comparable study estimates as a correction, in accordance with the Cochrane Handbook for Systematic Review of Interventions. ${ }^{48}$ In cluster RCTs, we will extract data that account for the clustering. If the trial accounted for cluster level effects by using multilevel modelling or generalised estimating equations, we will directly extract the data. If the study has not statistically accounted for cluster effects, we will use the generic inverse variance approach. The inverse variance approach weights studies to account for small samples, such as when studies are cluster randomised. ${ }^{54}$ Cluster analysis decisions will be made based on the number of clusters, outcome data accounting for cluster design and ICC by average cluster size. ${ }^{55}$

If data are missing, we will contact study authors to request the data where needed. We will attempt to use data from intention-to-treat (ITT) analyses. We will present ITT analyses for all primary outcomes using data corrected by mixed-effect models for repeated measures. If studies imputed missing data using last observation carried forward (LOCF), we will include them in the main 
meta-analysis but will exclude them during the sensitivity analysis due to uncertainty of reliability in LOCF methods.

\section{Statistical heterogeneity}

Given the potential heterogeneity between studies, we will conduct a random-effect pairwise meta-analysis for all analyses. A random-effect model assumes that the effects are different but sampled from a related representation of a larger population distribution, improving generalisability. ${ }^{46}$ We will assess statistical heterogeneity using CIs for individual trials visually represented in a forest plot. We will calculate the $I^{2}$ statistic with the suggested interpretation: $0 \%-40 \%$ may not be important; $30 \%-60 \%$ moderate heterogeneity; $50 \%-90 \%$ substantial heterogeneity; $75 \%-100 \%$ considerable heterogeneity. ${ }^{54}$ $I^{2}$ heterogeneity intervals overlap to allow for subjective decision-making that accounts for (1) effect magnitude and direction, (2) strength of evidence (ie, $p$ value size) and (3) detection of significant but very small effects in highly powered studies. ${ }^{54}$

\section{Assessment of reporting biases}

If the analysis contains 10 or more studies, data from included RCTs will be represented in a funnel plot to assess asymmetry. We will consider possible reasons of funnel plot asymmetry, including publication bias.

\section{Sensitivity analysis}

We will conduct sensitivity analyses to examine results robustness from main analyses. We will assess the effects of excluding studies with, and controls receiving more than usual care, studies using LOCF and studies with a high risk of bias in the areas of randomisation process, deviations from intended interventions, missing outcomes, measurement of outcome and selection of reported results.

\section{Subgroup analyses}

We will conduct subgroup analyses to examine differences between subsets of the population of interest and to understand heterogeneity. We will assess differences based on the following patient and trial characteristics: age, sex/gender, race/ethnicity, per cent insured, baseline severity and primary drug misused.

\section{Patient and public involvement}

No patient was involved in the protocol development nor will be involved in the review.

\section{Ethics and dissemination}

This study uses published aggregate data and does not require ethical approval. Findings will be disseminated in a peer-reviewed journal.

\section{Twitter Toshi A Furukawa @Toshi_FRKW}

Contributors ES and TAF conceived the study. ES, NW and TAF provided substantial contribution to the design of the study during its development. ES, MS, RT, CH, KY, YL, NW and TAF revised the study protocol critically for important intellectual content and contributed to its improvement. ES planned and conducted the search. TAF supervised the statistical analysis planning. ES, MS, RT, CH, KY, YL,
NW and TAF gave the final approval to publish the current protocol. All the authors agreed to be accountable for all aspects of the work in ensuring that questions related to the accuracy or integrity of any part of the work are appropriately investigated and resolved.

Funding This research was supported in part through a fellowship grant (P19110) and grant-in-aid (19F19110) awarded to ES and TAF, and a grant-in-aid (17H04122) awarded to TAF, from the Japan Society for the Promotion of Science.

Competing interests TAF reports personal fees from Mitsubishi-Tanabe, MSD and Shionogi, and a grant from Mitsubishi-Tanabe, outside the submitted work; TAF has a patent (2018-177688) pending. All the other authors report no competing interest.

Patient and public involvement Patients and/or the public were not involved in the design, conduct, reporting or dissemination plans of this research.

Patient consent for publication Not required.

Provenance and peer review Not commissioned; externally peer reviewed.

Open access This is an open access article distributed in accordance with the Creative Commons Attribution Non Commercial (CC BY-NC 4.0) license, which permits others to distribute, remix, adapt, build upon this work non-commercially, and license their derivative works on different terms, provided the original work is properly cited, appropriate credit is given, any changes made indicated, and the use is non-commercial. See: http://creativecommons.org/licenses/by-nc/4.0/.

ORCID iDs

Ethan Sahker http://orcid.org/0000-0002-4269-4800

Masatsugu Sakata http://orcid.org/0000-0002-5358-5263

\section{REFERENCES}

1 United Nations Office on Drugs and Crime. World drug report 2017: executive summary conclusions and policy implications. Vienna, Austria, 2017.

2 WHO. Lexicon of alcohol and drug terms published by the world Health organization. Terminol. Classif 2019.

3 American Psychiatric Association. Diagnostic and statistical manual of mental disorders: DSM-5. Washington, DC: American Psychiatric Association, 2013.

4 Han B, Gfroerer JC, Colliver JD, et al. Substance use disorder among older adults in the United States in 2020. Addiction 2009;104:88-96.

5 Han B, Hedden SL, Lipari R, et al. Receipt of services for behavioral health problems: results from the 2014 national survey on drug use and health, 2015. Available: http://www.samhsa.gov/data/sites/ default/files/NSDUH-DR-FRR3-2014/NSDUH-DR-FRR3-2014/ NSDUH-DR-FRR3-2014.htm

6 Substance Abuse and Mental Health Services Administration. Treatment episode data set - discharges (TEDS-D), 2017. Rockville, MD: Substance Abuse and Mental Health Services Administration, 2019.

7 Schildhaus S, Stocks C, Santora PB, et al. Community hospital admission from the emergency department by persons with substance use disorders. J Subst Abuse Treat 2013;44:201-7.

8 Dakour-Aridi H, Arora M, Nejim B, et al. Association between drug use and in-hospital outcomes after infrainguinal bypass for peripheral arterial occlusive disease. Ann Vasc Surg 2019;58:122-33.

9 Sahker E, Toussaint MN, Ramirez M, et al. Evaluating racial disparity in referral source and successful completion of substance abuse treatment. Addict Behav 2015;48:25-9.

10 Arndt S, Acion L, White K. How the states stack up: disparities in substance abuse outpatient treatment completion rates for minorities. Drug Alcohol Depend 2013;132:547-54.

11 Sahker E, Arndt S. Alcohol use screening and intervention by American primary care providers. Int J Drug Policy 2017;41:29-33.

12 Tran BX, Moir M, Latkin CA, et al. Global research mapping of substance use disorder and treatment 1971-2017: implications for priority setting. Subst Abuse Treat Prev Policy 2019;14:1-14.

13 Babor TF, Del Boca F, Bray JW. Screening, brief intervention and referral to treatment: implications of SAMHSA's SBIRT initiative for substance abuse policy and practice. Addiction 2017;112:110-7.

14 Cowell AJ, Dowd WN, Mills MJ, et al. Sustaining SBIRT in the wild: simulating revenues and costs for screening, brief intervention and referral to treatment programs. Addiction 2017;112:101-9.

15 Substance Abuse and Mental Health Services Administration. Grant awards archive FY 2001-2018, 2019. Available: https://www.samhsa. gov/grants/archive [Accessed 9 Oct 2019].

16 Barbosa C, Cowell A, Dowd W, et al. The cost-effectiveness of brief intervention versus brief treatment of screening, brief intervention 
and referral to treatment (SBIRT) in the United States. Addiction 2017;112:73-81.

17 Saitz R. Alcohol screening and brief intervention in primary care: absence of evidence for efficacy in people with dependence or very heavy drinking. Drug Alcohol Rev 2010;29:631-40.

18 Madras BK, Compton WM, Avula D, et al. Screening, brief interventions, referral to treatment (SBIRT) for illicit drug and alcohol use at multiple healthcare sites: comparison at intake and 6 months later. Drug Alcohol Depend 2009;99:280-95.

19 Saitz R. Screening and brief intervention for unhealthy drug use: little or no efficacy. Front Psychiatry 2014;5:1-5.

20 Glass JE, Hamilton AM, Powell BJ, et al. Specialty substance use disorder services following brief alcohol intervention: a meta-analysis of randomized controlled trials. Addiction 2015;110:1404-15.

21 Vendetti J, Gmyrek A, Damon D, et al. Screening, brief intervention and referral to treatment (SBIRT): implementation barriers, facilitators and model migration. Addiction 2017;112:23-33.

22 Saitz R, Alford DP, Bernstein J, et al. Screening and brief intervention for unhealthy drug use in primary care settings: randomized clinical trials are needed. J Addict Med 2010;4:123-30.

23 Young MM, Stevens A, Galipeau J, et al. Effectiveness of brief interventions as part of the screening, brief intervention and referral to treatment (SBIRT) model for reducing the nonmedical use of psychoactive substances: a systematic review. Syst Rev 2014;3:1-18.

24 Substance Abuse and Mental Health Services Administration. Systems-Level implementation of screening, brief intervention and referral to treatment, 2013.

25 Kaner EFS, Dickinson HO, Beyer F, et al. The effectiveness of brief alcohol interventions in primary care settings: a systematic review. Drug Alcohol Rev 2009;28:301-23.

26 Kaner EF, Beyer FR, Muirhead C, et al. Effectiveness of brief alcohol interventions in primary care populations. Cochrane Database Syst Rev 2018;2:CD004148.

27 Prochaska JO, DiClemente CC. Transtheoretical therapy: toward a more integrative model of change. Psychother Theory Res Pract 1982;19:276-88.

28 Rastegar D, Fingerhood M. The American society of addiction medicine handbook of addiction medicine. New York, NY: Oxford University Press, 2015.

29 Rollnick S, Miller WR. What is motivational interviewing? Behav Cogn Psychother 1995;23:325-34.

30 Miller WR, Zweben A, DiClemente CC, et al. Motivational enhancement therapy manual: a clinical research guide for therapists treating individuals with alcohol abus $E$ and dependence. Vol 2. Rockville, MD: National Institute on Alcohol Abuse and Alcoholism, 1995.

31 Society for Clinical Psychology APA. Psychological treatments. Motiv. interviewing, Motiv. Enhanc. ther. (MET), Met plus CBT mix. Subst. Abus, 2016. Available: https://www.div12.org/treatments/ [Accessed 30 Oct 2019].

32 Skinner HA. The drug abuse screening test. Addict Behav 1982;7:363-71.

33 Yudko E, Lozhkina O, Fouts A. A comprehensive review of the psychometric properties of the drug abuse screening test. J Subst Abuse Treat 2007;32:189-98.

34 National Institute on Drug Abuse. Instrument: drug abuse screening test (DAST-10), 2014. Available: https://cde.drugabuse.gov/ instrument/e9053390-ee9c-9140-e040-bb89ad433d69 [Accessed 17 Oct 2019].

35 Aldridge A, Linford R, Bray J. Substance use outcomes of patients served by a large US implementation of screening, brief intervention and referral to treatment (SBIRT). Addiction 2017;112:43-53.

36 US Preventive Services Task Force, Curry SJ, Krist AH, et al. Screening and behavioral counseling interventions to reduce unhealthy alcohol use in adolescents and adults: US preventive services Task force recommendation statement. JAMA 2018;320:1899-909.
37 Sahker E, Jones D, Lancianese D, et al. Racial/ethnic differences in alcohol and drug use outcomes following screening, brief intervention, and referral to treatment (SBIRT) in federally qualified health centers. J Racial Ethn Heal disparities 2019.

38 Bradley K, Berger D. Screening for unhealthy alcohol use. In: Saitz R ed. Addressing unhealthy alcohol use in primary care. New York, NY: Springer, 2013: 7-27.

39 U.S. Preventive Services Task Force. Final recommendation statement: drug use, illicit: screening, 2008. Available: https:// www.uspreventiveservicestaskforce.org/Page/Document/Reco mmendationStatementFinal/drug-use-illicit-screening [Accessed 9 Oct 2019].

40 Sahker E, Acion L, Arndt S. Age moderates the association of depressive symptoms and unhealthy alcohol use in the National guard. Addict Behav 2016;63:102-6.

41 Marsden J, Stillwell G, Barlow H, et al. An evaluation of a brief motivational intervention among young ecstasy and cocaine users: no effect on substance and alcohol use outcomes. Addiction 2006;101:1014-26.

42 Bashir K, King M, Ashworth M. Controlled evaluation of brief intervention by general practitioners to reduce chronic use of benzodiazepines. Br J Gen Pract 1994;44:408-12.

43 National Institutes of Health. Screening, brief intervention and referral to treatment (SBIRT) for drug abuse in general medical settings (R01). Req. APPL. number RFA-DA-08-021, 2008. Available: http:\%0A/grants.nih.gov/grants/guide/rfa-files/RFA-DA\%0A-08-021. html [Accessed 9 Oct 2019]

44 Moher D, Shamseer L, Clarke M, et al. Preferred reporting items for systematic review and meta-analysis protocols (PRISMA-P) 2015 statement. Syst Rev 2015;4:1-9.

45 Sobell LC, Sobell M, Buchan G, et al. Timeline followback method (drugs, cigarettes, and marijuana), 1996.

46 McLellan AT, Luborsky L, Woody GE, et al. An improved diagnostic evaluation instrument for substance abuse patients. The addiction severity index. J Nerv Ment Dis 1980;168:26-33.

47 WHO ASSIST Working Group. The alcohol, smoking and substance involvement screening test (ASSIST): development, reliability and feasibility. Addiction 2002;97:1183-94.

48 Higgins JPT, Thomas J, Chandler J, et al. Cochrane handbook for systematic reviews of interventions. 2 nd edn. Chichester, UK: The Cochrane Collaboration and John Wiley \& Sons Ltd, 2019.

49 Cohen J. Statistical power analysis for the behavioral sciences. New York, NY: Routledge, 1988.

50 Newcombe D, Tanielu-Stowers H, McDermott R, et al. The validation of the alcohol, smoking and substance involvement screening test (ASSIST) amongst Pacific people in New Zealand. NZ J Psychol 1994:45:30-9.

51 Khan R, Chatton A, Thorens G, et al. Validation of the French version of the alcohol, smoking and substance involvement screening test (ASSIST) in the elderly. Subst Abuse Treat Prev Policy 2012;7:14.

52 Khan R, Chatton A, Nallet A, et al. Validation of the French version of the alcohol, smoking and substance involvement screening test (ASSIST). Eur Addict Res 2011;17:190-7.

53 Rücker G, Cates CJ, Schwarzer G. Methods for including information from multi-arm trials in pairwise meta-analysis. Res Synth Methods 2017:8:392-403.

54 Deeks JJ, Higgins JPT, Altman DG, et al. Analysing data and undertaking meta-analyses. In: Higgins JPT, Thomas J, Chandler $\mathrm{J}$, et al, eds. Cochrane handbook for systematic reviews of interventions. Chichester, UK: The Cochrane Collaboration and John Wiley \& Sons Ltd, 2019: 241-84.

55 Higgins JPT, Eldridge S, Li T, et al. Including variants on randomized trials. In: Higgins JPT, Thomas J, Chandler J, et al, eds. Cochrane Handbook for systematic reviews of interventions. Chichester, UK: The Cochrane Collaboration and John Wiley \& Sons Ltd, 2019: 569-93.

56 Cheung MW-L, Ho RCM, Lim Y, et al. Conducting a meta-analysis: basics and good practices. Int J Rheum Dis 2012;15:129-35. 